\title{
Large-scale motion, oscillations and a possible halo on the counter-jet side in $1803+784$
}

\author{
S. Britzen ${ }^{1,2,3}$, T. P. Krichbaum ${ }^{1}$, R. G. Strom ${ }^{3,4}$, A. Witzel $^{1}$, T. W. B. Muxlow ${ }^{5}$, L. I. Matveenko ${ }^{6}$, R. M. Campbell ${ }^{7}$, \\ W. Alef ${ }^{1}$, C. A. Hummel ${ }^{8,9}$, and A. Zensus ${ }^{1}$
}

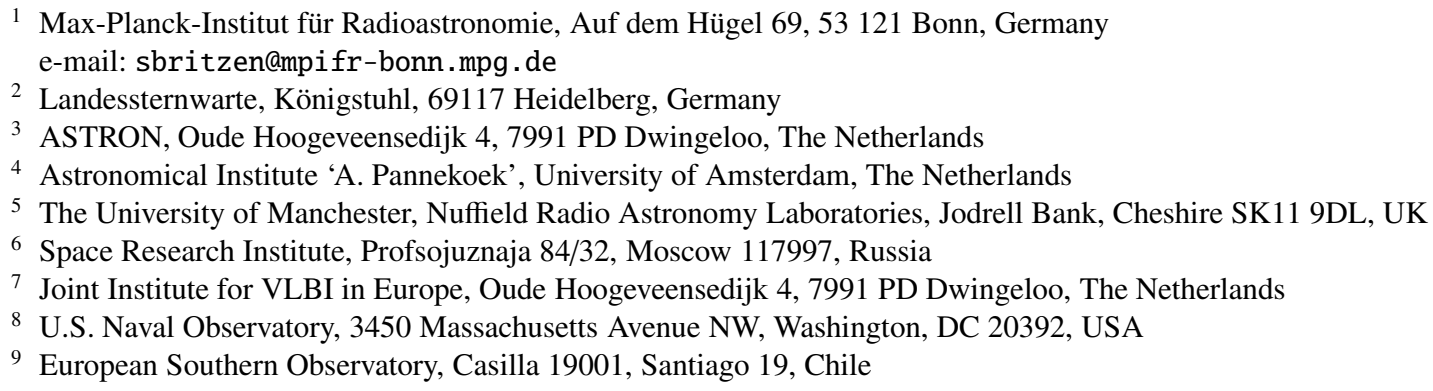

Received 10 August 2004 / Accepted 23 August 2005

\section{ABSTRACT}

\begin{abstract}
We present world-array VLBI observations of the blazar 1803+784 performed on May 29, 1993 at $\lambda=18 \mathrm{~cm}$. A 17-station VLBI array, the phased VLA and the 7-station MERLIN array observed the source simultaneously for $11 \mathrm{~h}$. We present the global VLBI map, the MERLIN map, and combined-array maps at different resolutions giving an overview of the morphology of $1803+784$ from the inner 10 mas to $\sim 2$ arcsec. We show that the jet bends by around $90^{\circ}$ at a core separation of about 0.5 arcsec towards the South. On larger scales, a Westerbork Synthesis Radio Telescope (WSRT) map made at a wavelength of $6 \mathrm{~cm}$ resolves the bridge connecting the core with the secondary component some 45" to the south. Wiggles in the ridge of this emission suggest that, as on the 100-pc scale, the jet may also oscillate on 50-100 kpc scales. In addition to improved imaging of the southern component, the $6 \mathrm{~cm}$ map provides evidence for amorphous emission to the north, as well as an extended, halo-like component about the nucleus. We compare the source structure at 6 and $18 \mathrm{~cm}$ with the structure at $2 \mathrm{~cm}$ in a VLBA image obtained on November 6, 1999. Jet wiggling is clearly seen on all scales between 1 mas and 50 arcsec. We find some indication for apparent superluminal motion between $\sim 5 c$ and $7 c$ for the 25 mas jet component in model-fit results for five epochs of VLBI observations performed at 6 and $18 \mathrm{~cm}$. In addition, we find some evidence for further so-called stationary components in the jet of this source which will have to be confirmed in future observations spaced more densely in time. We discuss the self-similar source morphology observed from mm to $\mathrm{cm}$ wavelengths - namely the wiggling on all observed scales - and the implications of the deviations from self-similarity. This is the second of two papers describing the radio structure of S5 $1803+784$. The first presented the results of 6 years of VLBI monitoring in the $X$-band (Britzen et al. 2005, MNRAS, in press).
\end{abstract}

Key words. techniques: interferometric - BL Lacertae objects: individual: 1803+784 - radio continuum: galaxies

\section{Introduction}

The optical object associated with $1803+784$ is a $16.4 \mathrm{mag}$ BL Lacertae object (BL Lac) with a redshift of 0.68 (Lawrence et al. 1987; Stickel et al. 1993) and, unusually for BL Lacs, with particularly strong emission lines (Lawrence et al. 1987). The problem of "BL Lac objects with strong emission lines" has been addressed by several authors (e.g., Antonucci et al. 1987; Kollgaard et al. 1992; Stickel et al. 1991). In order to avoid the inevitable confusion of taxonomy we will call this object a blazar hereafter. In this paper we use the following cosmological parameters: $H_{0}=65 \mathrm{~km} \mathrm{~s}^{-1} \mathrm{Mpc}^{-1}, q_{0}=0.5$.
At the distance of $1803+784,1$ mas corresponds to a length of $6.08 \mathrm{pc}$ projected on the plane of the sky.

A number of other nearby radio and X-ray sources (Biermann et al. 1992) are probably unrelated (Johnston et al. 1984). This source is a member of the complete S5 sample of 13 flat-spectrum radio sources (Witzel 1987). The sources of this sample have been observed repeatedly at many frequencies and angular resolutions since the late 1970's. The complex morphology of the mas-jet of $1803+784$ has been studied in VLBI observations at different radio wavelengths (e.g., Eckart et al. 1986, 1987; Witzel et al. 1988). 
$1803+784$ reveals peculiar properties that have been investigated by a number of authors, e.g., Strom \& Biermann (1991), Gabuzda (1999), Gabuzda \& Cawthorne (2000), Gabuzda \& Chernetskii (2003), Ros et al. (2000, 2001). As an intraday variable, the source shows rapid flux-density variations in the optical and radio regime (Wagner \& Witzel 1995) on timescales as short as $50 \mathrm{~min}$ in the optical (Wagner et al. 1990). Jet components move with apparent superluminal velocities on strongly curved trajectories that can be traced with mmVLBI to the innermost regions of the AGN (e.g., Krichbaum 1990; Krichbaum et al. 1993). The coexistence of superluminally moving and oscillating components has been deduced from VLBI monitoring performed at $3.6 \mathrm{~cm}$ wavelength by Britzen \& Krichbaum (1995) and Britzen et al. (2005), the latter paper also presenting a detailed discussion of the pc-scale radio-jet properties.

Another remarkable property of $1803+784$ is the difference in position angle between parsec- and kiloparsec-scale jets, placing it in the population of orthogonally misaligned objects. Pearson \& Readhead (1988) found in the distribution of $\triangle$ PAs a highly unexpected bimodal distribution of relatively well aligned and roughly orthogonal jets. The so-called "misaligned population" of core-dominated AGN reveals a $\triangle \mathrm{PA}$ of $70^{\circ}$ to $90^{\circ}$ (which is the so-called "secondary peak" in Appl et al. 1996).

More current investigations of larger samples by e.g., Wehrle et al. (1992), Conway \& Murphy (1993), and Appl et al. (1996), proved the secondary-peak sources to be statistically significant compared to the predictions of a single population simple bend model. Global VLBI observations reveal the pc-scale jet of $1803+784$ to be oriented in the East-West direction (Britzen et al. 2005), while the large-scale structure comprises a dominant core component and a weak secondary component $\sim 45^{\prime \prime}$ away from the core at position angle $\Theta \approx-166^{\circ}$ south-south-west, which is presumably physically related to the blazar (Antonucci et al. 1986). The connection between the pcand kpc-scale jet remained unclear for quite some time and observations presented in this paper bear directly on this matter. In only a few similar cases have VLBI maps been able to trace the jets in the transition region, and hence to determine the speed, expansion, and accompanying changes in flux density along the jet (e.g., Benson et al. 1988; Muxlow et al. 1988; Hummel et al. 1992). In order to study the jet of $1803+784$ and its curved appearance at high resolution over its entire length and to detect the bending towards the southern kpc-component, we used a combined global VLBI-MERLIN array. $1803+784$ is one of the largest one-sided radio sources known (Strom \& Biermann 1991).

Section 2 gives an overview of the radio structure of the jet of $1803+784$ on pc- and kpc-scales. Section 3 then summarizes the details of the observations $(18 \mathrm{~cm}$ world-array, $6 \mathrm{~cm}$ WSRT, $6 \mathrm{~cm}$ VLBA+Eb and VLBA, and $2 \mathrm{~cm}$ VLBA) and the data reduction. Sections 4 and 5 present the maps at different angular resolutions, trace the jet structure in these maps, search for jet component motion in the jets at larger core separations ( $\sim 25$ mas) by model fitting, and discuss the results. Preliminary results have already been presented in Britzen et al. (1999) and Britzen (2002).

\section{The radio jet of $1803+784$}

\subsection{The pc-scale jet}

On pc-scales the source shows a pronounced jet, with prominent jet components located at 1.4, 5, and 12 mas from the core (Eckart et al. 1986). Geodetic and astronomical VLBI data obtained at various epochs between 1979 and 1985 indicated that the component at 1.4 mas is stationary (Schalinski et al. 1988; Witzel et al. 1988). Several authors investigated this source (Charlot 1990; Fey et al. 1996; Kellermann et al. 1998); some confirm the constant separation (e.g., Cawthorne et al. 1993). Britzen et al. (2005) give a detailed analysis of the smallscale radio structure of the jet of $1803+784$ as observed in $X$-band monitoring and discuss significant position shifts observed for the brightest jet component with displacements between $r \sim 0.7$ mas and $r \sim 1.5$ mas. Obviously the improved time sampling of the geodetic VLBI data led to the detection of systematic position variations for components regarded as stationary on the basis of the less frequently targeted astronomical observations. This oscillatory behaviour is explained as the result of a reconfinement shock (Britzen et al. 2005). In addition, superluminal motion has been detected in VLBI observations at 43, 22, (Krichbaum et al. 1994) and 8.4 GHz (Britzen et al. 2005). VLBI observations at $43 \mathrm{GHz}$ revealed for the first time evidence for a jet that has been called "helical" by Krichbaum (1990). Such curved-jet morphology is found also at larger core separations from VLBI maps obtained at $S$ - and $X$-band (Britzen \& Krichbaum 1995; Britzen et al. 2005).

\subsection{The kpc-scale jet}

Observations at $1.5 \mathrm{GHz}$ by Antonucci et al. (1986) found a $2^{\prime \prime}$ extension west and south of the core at a position angle of $\Theta \approx-120^{\circ}$, as well as a weak secondary component $\sim 45^{\prime \prime}$ away at a position angle of $\Theta \approx-166^{\circ}$ south-south-west of the core. Strom \& Biermann (1991) also detected this secondary component, as well as a bridge connecting it with the core, with 1.5 GHz WSRT observations. Kollgaard et al. (1992) observed $1803+784$ with both the D and B arrays of the VLA. Lowresolution images show the core and secondary component, neither of which is resolved. No extended emission was detected around the core. The secondary is best seen in the highresolution data on a highly tapered map, and appears to extend back towards the core. Although Strom \& Biermann (1991) refer to the secondary as a "hotspot", the B-array observations by Kollgaard et al. (1992) show that it is not compact. However, none of these observations showed the transition where the bending from the pc-jet to the kpc southern component takes place.

\section{Observations and data reduction}

\section{1. $2 \mathrm{~cm} \mathrm{VLBI}$ observations}

The VLBA observations of this source took place on November 6, 1999 and were provided by E. Ros. The data have been edited, reduced and calibrated within the work on the $2 \mathrm{~cm}$ survey (Kellermann et al. 2004). We performed model fitting 
within the difmap-package by fitting Gaussian model components directly to the observed visibilities (real and imaginary parts) using the Levenberg-Marquardt non-linear least squares minimization technique (program modelfit within difmap) to fit the brightness, sizes and positions of the individual jet components.

\section{2. $6 \mathrm{~cm} V L B A+E b$ observations}

$5 \mathrm{GHz}$ VLBA plus Effelsberg observations were performed on September 5, 1999. Data calibration and analysis was performed in the standard way: the data were fringe fitted and averaged within AIPS. After calibration we obtained a Clean map and modelfit within the difmap package.

\section{3. $18 \mathrm{~cm}$ world-array VLBI observations}

1803+784 was observed on 29 May 1993 simultaneously with a world-array of telescopes in MK II mode at $1.66 \mathrm{GHz}$ for $11 \mathrm{~h}$. The telescopes (plus their countries and diameters [in m]) were: Onsala (Sweden, 25); Effelsberg (Germany, 100); WSRT (Netherlands, phased array, 93); Cambridge (United Kingdom, 18), Jodrell Bank 1 (76), Knockin (25); Evpatoria (Russia, 64), Bearlake (64); Bologna (Italy, 32), Noto (32); Shanghai (China, 25); VLA (USA, phased array) and the ten VLBA antennas (USA, each 25). In addition to these telescopes, a local interferometer, namely the MERLIN array, participated in the observations. The MERLIN array added baselines in the range $20-1200 \mathrm{k} \lambda$ to the inner part of the uv coverage of the VLBI array. At some of the telescopes, problems occurred during the observations (e.g., Mauna Kea). We removed those data that could not be calibrated well enough from the final data set. The uv coverage obtained with the remaining array is shown in Fig. 1. The VLBI and MERLIN maps were correlated, fringe-fitted and calibrated separately, and combined afterwards.

\subsubsection{Global VLBI data reduction}

The VLBI data were recorded in MK II mode and correlated with the 16-station MK II correlator at CalTech in Pasadena under supervision of S. Unwin. The dataset was split into two parts and each was correlated separately. After correlation, the data were processed at the Max-Planck-Institut für Radioastronomie in Bonn. The data were imported into the AIPS package, and the two separately correlated data sets were added using AIPS tasks UVCOP and DBCON to form one complete data set. The fringe fitting of the complete data set was done within AIPS. After fringe fitting the data were averaged (two minutes) and exported into the CIT package where the calibration was done. Imaging was performed within the difmap package afterwards.

\subsubsection{MERLIN data reduction}

The MERLIN data reduction as well as the combination of the MERLIN and VLBI world-array map was done using the

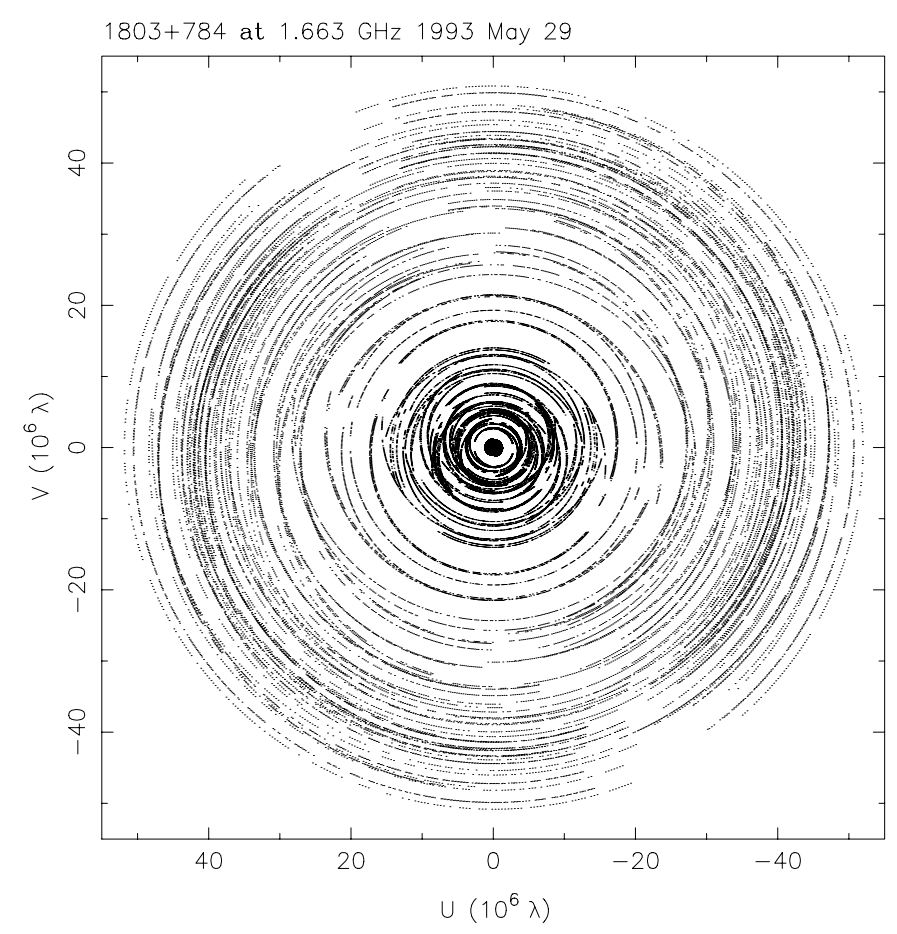

Fig. 1. uv coverage obtained with the full array of VLB antennas (see text) for the world-array observations.

OLAF and AIPS package at Jodrell Bank. The data were exported from AIPS in FITS format and converted to OLAF with the routine RFITS. The OLAF package is especially suited to perform very high dynamic-range continuum imaging (Muxlow et al. 1988; MERLIN User Guide, Version 3, Jan. 2003). The VLBI global array map was used as the initial model for the MERLIN-VLBI combined array to self-calibrate the complete dataset. Special care had to be taken to modify the weights of the individual antennas.

\section{4. $6 \mathrm{~cm}$ WSRT observations}

The Westerbork Synthesis Radio Telescope (WSRT) observed $1803+784$ at a wavelength of $6 \mathrm{~cm}$ on 2/3 August 2000 in standard synthesis mode. The eight $10 \mathrm{MHz}$ contiguous bands of the digital correlator were centered at a sky frequency of $4839 \mathrm{MHz}$. The four movable elements of the array were set to a minimum redundancy configuration ("maxi-short"), and the observation lasted for $12 \mathrm{~h}$, providing a beamwidth of $3.4^{\prime \prime} \times 3.5^{\prime \prime}$ and a noise level of under $50 \mu \mathrm{Jy} / \mathrm{beam}$. The first grating response at a distance of $1.6^{\prime}$ has a size substantially greater than that of the entire source.

The data were calibrated for gain in a standard way (Windhorst et al. 1984), and subsequent self-calibration was applied iteratively, using clean component models of the extended emission, to obtain a map with a dynamic range of nearly $50 \mathrm{~dB}$. As discussed below, the structure seen in the final map is consistent with previous published work. 


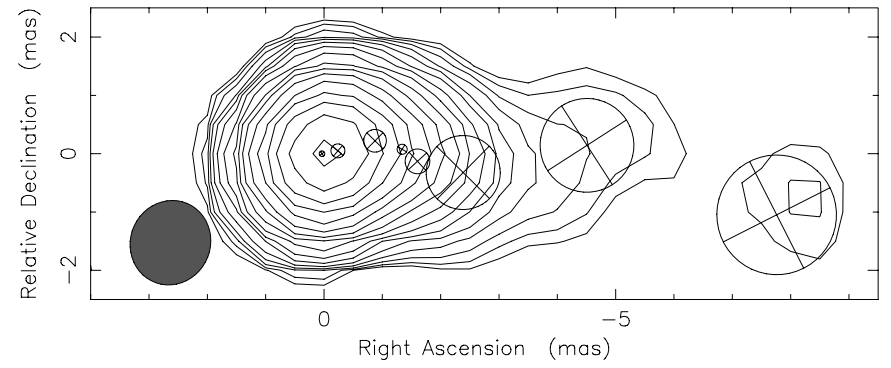

Fig. 2. A model fit convolved with the interferometric beam and with the residual map added is shown with the Gaussian model components superimposed. The $2 \mathrm{~cm}$ data were obtained with the VLBA on November 6, 1999 (Kellermann et al. 2004). The map peak is $2.06 \mathrm{Jy} /$ beam. The contour levels shown start at $0.2 \%$. The scale factor between subsequent levels is 1.5 .

\section{Results}

\subsection{The morphology obtained in VLBA, world-array $V L B I$, and WSRT observations}

In Fig. 2, we show a model fit performed from observations obtained at $2 \mathrm{~cm}$ (Kellermann et al. 2004). Figures 3a-c, and 4 show a sequence of Clean maps originating from the worldarray VLBI observations and MERLIN. To study the morphology of $1803+784$ at different angular resolutions we imaged the corresponding parts of the uv-dataset separately. In Fig. 5 we show the source structure obtained in observations with the WSRT. In the following we give an overview of the structure of $1803+784$ at different angular resolutions:

\subsubsection{The inner 10 mas}

Figure 2 shows the inner 10 mas of the jet structure of $1803+784$. Clearly visible is a chain of jet components that follows a curved jet structure, suggesting at least three bends within the inner 8 mas of the jet. This jet structure is typical and has been traced at $\mathrm{mm}$ - and cm-wavelengths for almost three decades (e.g., Krichbaum et al. 1990; Britzen et al. 2005).

\subsubsection{The inner 50 mas}

The map shown in Fig. 3a (beam: $2.708 \times 2.844$ mas, PA: $27.55^{\circ}$ ) results from the global VLBI array alone. This is the highest angular resolution image based on the worldarray dataset. The source shows an east-west directed jet with a bright jet component at a core separation of $\sim 25$ mas which shows obvious substructure and has a sharp edge towards the direction of the core. Downstream from this prominent jet feature the jet "exhausts", bends northward and becomes less confined.

\subsubsection{The inner 100 mas}

The following maps (Figs. 3b,c) are combined array $($ MERLIN + global VLBI) maps at medium resolution and have been obtained by mapping the equivalent parts of the uvdata sets. The beam in Fig. 3 b is $8.41 \times 7.33$ mas with a position angle of $-39.3^{\circ}$. On these scales the radio structure is directed mainly in the east-west direction. A bend towards the north (at $~ 30$ mas separation from the core) is followed by a significant bend towards the south (Fig. 3b), another bend towards the north follows (Fig. 3c). Obviously the oscillating jet structure seen in VLBI observations at higher frequencies (Fig. 2) between 0.5 and 8 mas continues in the $18-\mathrm{cm}$ "combined array" maps to $\sim 60$ mas, making the jet of $1803+784$ an example of a self-similar jet.

\subsubsection{Arcseconds}

Figure 4 shows the large scale structure of $1803+784$ seen with the MERLIN-only array (beam: $0.28949 \times 0.23594$ arcsec, PA: $-2.46^{\circ}$; dynamic range: $\left.\sim 14000: 1\right)$. The map shows the core and a jet extending towards the west that bends towards the south at a separation of $\sim 0.5$ arcsec. This bending most likely marks the change in orientation between the direction of the pc- and the kpc-scale jet. The jet structure at this resolution is extremely weak and can only be detected in the high dynamic-range observations performed with MERLIN.

The jet oscillation is significant on these comparatively large scales as well and is obviously not disturbed by the bending of the overall jet structure towards the South. The curved jet can be traced over $\sim 2$ arcsec length to the South in these observations.

\subsubsection{Large-scale structure}

Figure 5 shows the bridge linking the core with the secondary component to the south in a Clean map obtained from observations with the WSRT. This structure is consistent with the VLA maps published by Kollgaard et al. (1992). In their $\mathrm{B}$-array image, the peak of the southern extension has a brightness comparable to that of the WSRT map. No emission is seen around the nucleus in their map, to a limit of $0.525 \mathrm{mJy} / \mathrm{beam}$ (or $80 \mu \mathrm{Jy} \operatorname{arcsec}^{-2}$ ), while the brightest peak in their Fig. 3, roughly $15^{\prime \prime}$ south of the nucleus, has a brightness of only $0.15 \mathrm{mJy} / \mathrm{beam}\left(14 \mu \mathrm{Jy} \operatorname{arcsec}^{-2}\right)$. We further note that the total flux density of the southern extension from the Kolgaard et al. D-array map agrees well with that expected from the previous Strom \& Biermann (1991) WSRT data at lower frequencies. The resulting spectral index, from 6 to $49 \mathrm{~cm}$, is $\alpha=-1.0$ (with $S(v) \propto v^{-\alpha}$ ).

In addition to the southern extension, our WSRT $6 \mathrm{~cm}$ map suggests there is other emission surrounding the core. At the lowest intensity levels there is some distortion by calibration effects (the two indentations seen on the lowest contour west and northwest of the core are where east-west linear depressions cut through the source emission). Nonetheless, we believe that there is halo-like emission around the central component, with 


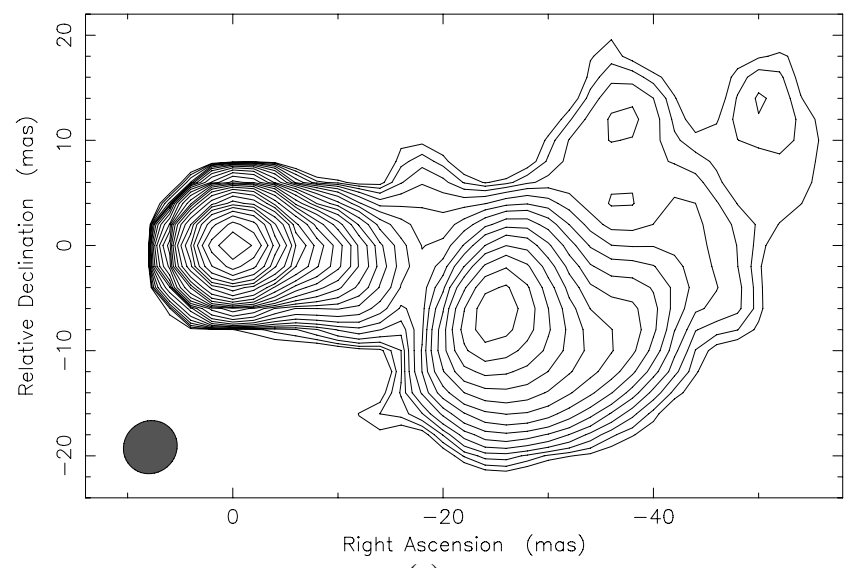

(a)

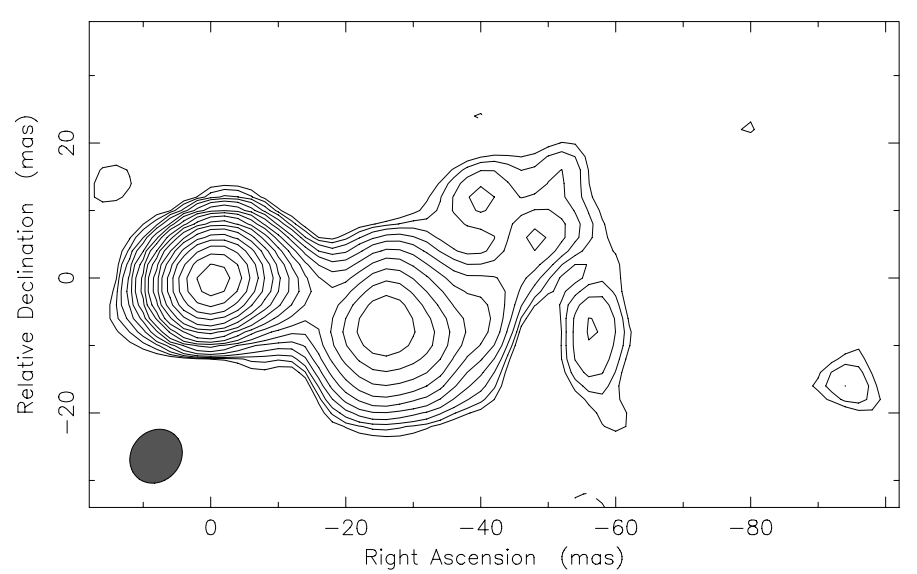

(b)

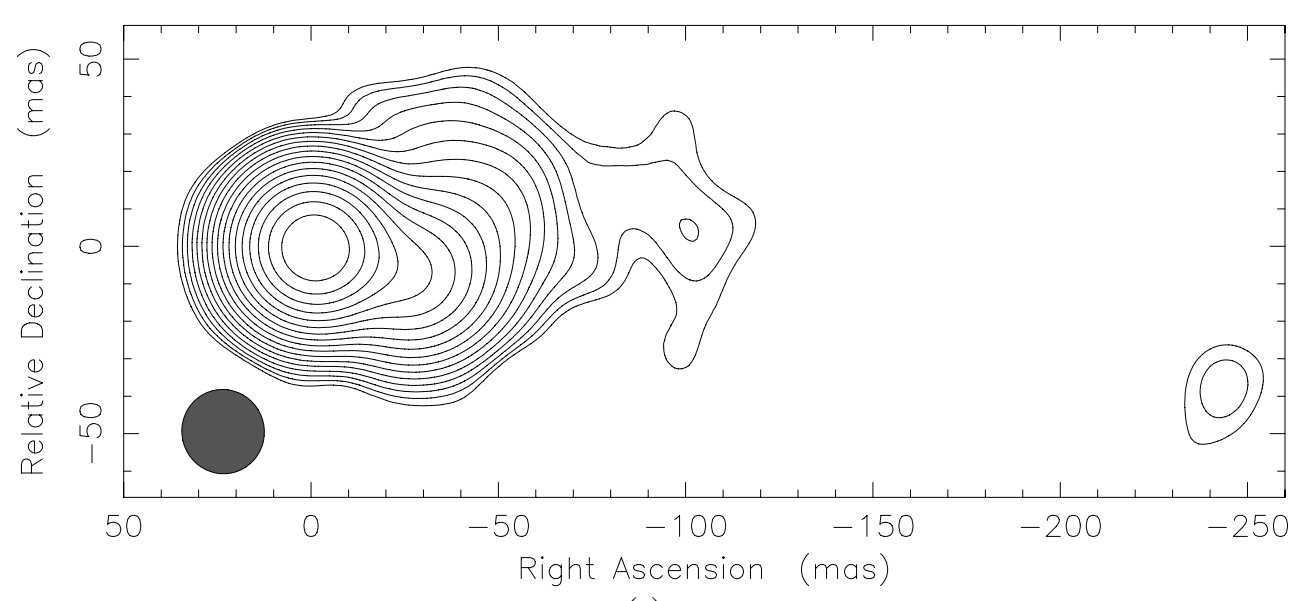

(c)

Fig. 3. Three VLBI images of $1803+784$ at $18 \mathrm{~cm}$ wavelength in order of decreasing angular resolution (from top to bottom; a) to c)). Global VLBI a), and combined array maps (b), c)) of $1803+784$ at $18 \mathrm{~cm}$ wavelength. The map peak in the images is $1.36 \mathrm{Jy} / \mathrm{beam}, 1.44 \mathrm{Jy} / \mathrm{beam}$, and $1.62 \mathrm{Jy} /$ beam, in a) $-\mathbf{c}$ ) respectively. The $F W H M$ beamsize is shown in the lower left corner and corresponds to $5.22 \times 4.94 \mathrm{mas}$ at $-51.3^{\circ}$ in a), $8.41 \times 7.33$ mas at $-39.3^{\circ}$ in $\mathbf{b}$ ), and $22.5 \times 22$ mas at $14.9^{\circ}$ in $\mathbf{c}$ ). Contour values are in steps of 1.3 from 0.11 to 77.6 in a), in steps of 1.5 from -0.12 to 78.8 in $\mathbf{b}$ ), and in steps of 1.5 from 0.1 to 65.7 in $\mathbf{c}$ ).

a secondary peak $\sim 20^{\prime \prime}$ north of it. If this is the counterpart to the southernmost peak, then the brightness contrast is $15: 1$.

\subsubsection{Summary of structure over all scales}

Summarizing the structural information for $1803+784$ probing different scales in its jet, we find at $\sim 25$ mas a prominent jet feature from where the jet turns towards the north. Largerscale maps at $18 \mathrm{~cm}$ show that the jet continues to wiggle in an East-West direction farther out, and although the jet is bent and redirected towards the South at a core separation of $\sim 0.5^{\prime \prime}$, jet wiggling is not interrupted and is still pronounced even on these large scales. The direction of the jet towards the South shown in the largest scale map of the MERLIN observations (Fig. 4) agrees well with the general direction of the jet in the WSRT map (Fig. 5). Both maps again show significant evidence for a continuation of the oscillations on kpc-scales. The WSRTmap nicely resolves the so-called bridge between the core of $1803+784$ and the southern component. In addition, this map shows evidence for halo-like emission around the central component with a secondary peak north of it.

\subsection{Search for Motion of Jet components in the intermediate-scale structure}

In Table 1 we give the model-fit parameters for five analyzed VLBI data sets from different epochs. Column 1 denotes the epoch of the observation, Col. 2 the wavelength, Col. 3 the component identification (using the nomenclature directly from the literature, where appropriate), Col. 4 the flux-density $(S)$ of the jet component, Col. 5 the core separation $(r)$, Col. 6 the position angle of the center of the jet component $(\Theta)$, Col. 7 the major axis, Col. 8 the ratio of the minor axis to the major axis, Col. 9 the position angle of the major axis $(\Phi)$, and Col. 10 the VLBI array and references for the data. Two data sets were taken from the literature (Eckart et al. 1987 and Aaron 1996). The other two epochs are the global-VLBI observations of this paper and VLBA data (as calibrator source) obtained within multi-frequency VLBI observations of 3C 390.3 in 1993.96 


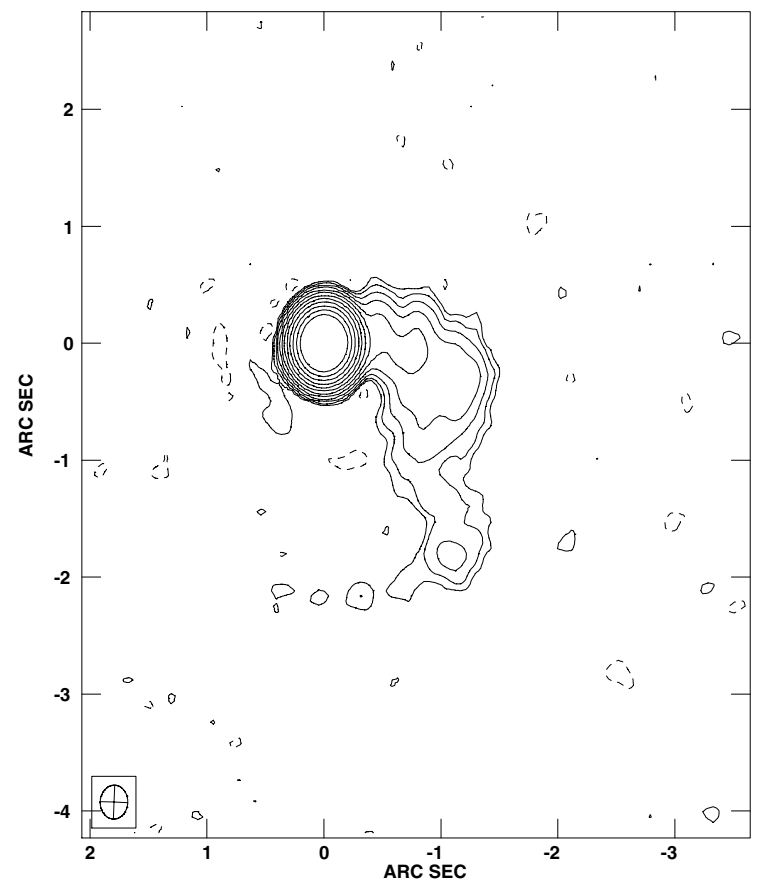

Fig. 4. Map obtained with the data from the MERLIN array only. This is the largest scale map obtained from the world-array observations. The peak flux is $1.9643 \mathrm{Jy} /$ beam. The contour levels shown correspond to $0.0001375 \times(-1,1,2,4,8,16,32,64,128,256,512,1024$, 2048). The $F W H M$ beamsize is shown in the lower left hand corner.

(Preuss et al. 1996). Two epochs are $18 \mathrm{~cm}$ VLBA observations (1993.96, 1995.79), while the other two $18 \mathrm{~cm}$ data sets result from global VLBI observations (1981.79, 1993.41). In addition to these four $18 \mathrm{~cm}$ data-sets, we also list the Gaussian component parameters for a $6 \mathrm{~cm}$ observation. We admit that difficulties occur naturally when comparing results obtained from datasets taken 14 years apart. The difference in data quality usually affects the number of jet components that can be fit. The number of detected jet components in the four analyzed epochs is different. The three jet components detected by Eckart et al. (1987) can be traced through the epochs, while the components at larger core separations can only be seen in a subsample of the more recent data sets. Three data sets in addition yield evidence for a component at a core separation of $\leq 3$ mas.

To compensate for the smaller number of jet components of the dataset observed in 1983 we performed the model-fitting of the (world-array) global VLBI dataset twice with different numbers of jet components, in order to enable a comparison of the results for the different epochs.

The error analysis has to take differences in data quality and resolution of the data sets into account. The errors of epoch 1993.41 and 1993.96 were determined by comparing the results of several model-fitting runs performed when including different numbers of components. For the uncertainties of the $6 \mathrm{~cm}$ data we take: $\Delta S: 10 \% ; \Delta r: 0.2$ mas for the components at $r<28$ mas and $\Delta r: 1$ mas for the component at $28 \mathrm{mas} ; \Delta \Theta: 5^{\circ}$.

In our further analysis we concentrate on the brightest jet components within 30 mas of the core and indicate this in Table 1 by separating the components by the first horizontal

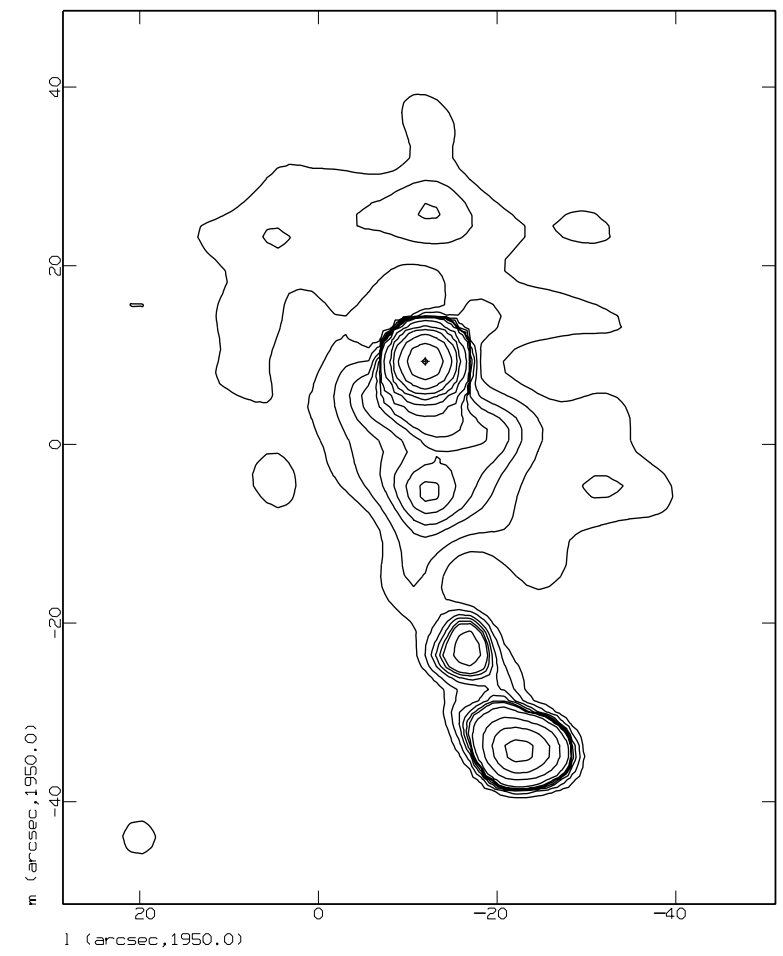

Fig. 5. Clean map of $1803+784$ obtained with the WSRT at $6 \mathrm{~cm}$ (August 2000). Contour values are 0.035, 0.065, 0.095, 0.115, 0.135, $0.15,0.2,0.4,0.8,1.5,3,7$, and $14 \mathrm{mJy} /$ beam. $99 \%$ of the flux density from the nuclear component has been subtracted from this map. The $F W H M$ beamsize is shown in the lower left hand corner.

dotted line. In Fig. 6 we show a model fit convolved with the interferometric beam and with the residual map added $(5 \mathrm{GHz}$ VLBA observations, September 1999) as an example of the model fits performed. We trace several jet components and identify four of them at core separations between 5 and 30 mas. The observations from 1993.41 on allow us to detect substructure in the $r \sim 25$ mas component. This component is a blend of two jet components, one located at $\sim 25$ mas, the other at $\sim 28$ mas core separation. For our analysis we consider three different scenarios: we can assume that the 24.8 mas component seen by Eckart et al. (1987) is identical with the $r \sim 25$ mas component seen in the other epochs. In this scenario we assume that the $r \sim 28$ mas component was not visible in Eckart et al. ("one to one" scenario in Table 2: case I). Another possibility is to assume that the 24.8 mas component seen by Eckart et al. (1987) is a blend of the two components which can be detected in the more recently performed observations ("one to two" scenario in Table 2, case II). The position of the blended component in the later epochs is taken to be the mean position of the two components (not listed in Table 1). The second scenario is supported by the flux-density value of the $r \sim 25$ mas component seen by Eckart et al. (1987) (see Fig. 8), which is considerably higher than the $r \sim 25$ mas component seen in the other VLBI observations. In the third scenario, we assume the "one to two" scenario but perform a flux-density weighted calculation to determine the position of the blended component in Table 1 between the horizontal dotted lines (case III in Table 2). This solution seems to 
Table 1. Modelfit parameters and uncertainties for four $\lambda=18 \mathrm{~cm}$ and one $\lambda=6 \mathrm{~cm}$ dataset (see text for details concerning the data).

\begin{tabular}{|c|c|c|c|c|c|c|c|c|c|}
\hline Epoch & $\begin{array}{c}\lambda \\
{[\mathrm{cm}]}\end{array}$ & Comp. & $\begin{array}{c}S \\
{[\mathrm{Jy}]}\end{array}$ & $\begin{array}{c}r \\
{[\mathrm{mas}]}\end{array}$ & $\begin{array}{c}\Theta \\
{[\operatorname{deg}]}\end{array}$ & $\begin{array}{c}\text { Maj. ax. } \\
\text { [mas] }\end{array}$ & Ratio & $\begin{array}{c}\Phi \\
{[\mathrm{deg}]}\end{array}$ & $\begin{array}{l}\text { Ref. } \\
\text { Array }\end{array}$ \\
\hline \multirow[t]{5}{*}{1981.79} & 18 & L1 & $1.29 \pm 0.13$ & 0.0 & 0.0 & $<2.0$ & $>0.50$ & & Eckart et al. $(1986,1987)$ \\
\hline & & $\mathrm{L} 2$ & $0.25 \pm 0.03$ & $6.1 \pm 1.6$ & $248 \pm 14$ & $4.7 \pm 1.5$ & $>0.29$ & $60 \pm 20$ & Crimea, Dwingeloo, \\
\hline & & L3 & $0.15 \pm 0.02$ & $11.8 \pm 1.1$ & $258 \pm 5$ & $<4$ & $>0.25$ & & MPIfR, Jodrell Bank, \\
\hline & & L4 & $0.20 \pm 0.02$ & $24.8 \pm 1.8$ & $261 \pm 4$ & $<9$ & $>0.11$ & & NRL (Washington), OVRO \\
\hline & & & & & & & & & Onsala, NRAO (Green Bank) \\
\hline \multirow[t]{12}{*}{1993.41} & 18 & $\mathrm{C} 1$ & $1.387 \pm 0.267$ & 0.0 & 0.0 & $1.71 \pm 0.18$ & $0.07 \pm 0.22$ & $89.78 \pm 5.2$ & this paper \\
\hline & & $\mathrm{K} 1$ & $0.078 \pm 0.022$ & $2.82 \pm 0.11$ & $-94.01 \pm 2.81$ & $2.96 \pm 0.50$ & $<0.01$ & $-13.37 \pm 0.18$ & world-array \\
\hline & & $\mathrm{K} 2$ & $0.151 \pm 0.021$ & $5.52 \pm 0.09$ & $-96.29 \pm 5.24$ & $5.43 \pm 0.70$ & $0.38 \pm 0.12$ & $86.70 \pm 1.87$ & \\
\hline & & $\mathrm{K} 3$ & $0.047 \pm 0.006$ & $10.79 \pm 0.22$ & $-104.01 \pm 1.63$ & $7.20 \pm 0.59$ & $0.75 \pm 0.25$ & $64.60 \pm 12$ & \\
\hline & & K4 & $0.056 \pm 0.004$ & $25.01 \pm 0.10$ & $-103.98 \pm 0.85$ & $8.73 \pm 0.54$ & $0.23 \pm 0.02$ & $-23.51 \pm 1.96$ & \\
\hline & & K5 & $0.183 \pm 0.007$ & $27.95 \pm 1.32$ & $-106.35 \pm 0.42$ & $12.68 \pm 0.9$ & $0.71 \pm 0.16$ & $-11.52 \pm 0.67$ & \\
\hline & & & ………......... & ……........ & . & $\ldots \ldots \ldots \ldots . .$. & $\ldots$ & ……............. & \\
\hline & & $\mathrm{K} 4 / 5$ & & 27.26 & & & & & \\
\hline & & K6 & $0.008 \pm 0.004$ & $17.61 \pm 2.09$ & $-79.24 \pm 6.73$ & $10.63 \pm 2.0$ & $0.40 \pm 0.25$ & -24.96 & \\
\hline & & $\mathrm{K} 7$ & $0.029 \pm 0.019$ & $35.02 \pm 0.89$ & $-66.06 \pm 7.8$ & $34.81 \pm 15$ & $0.35 \pm 0.13$ & 22.28 & \\
\hline & & K8 & $0.037 \pm 0.002$ & $38.30 \pm 0.23$ & $-104.38 \pm 1.23$ & $13.49 \pm 0.16$ & $0.43 \pm 0.02$ & $-19.09 \pm 2$ & \\
\hline & & K9 & $0.043 \pm 0.002$ & $48.63 \pm 1.02$ & $-80.79 \pm 4.74$ & $27.38 \pm 2.20$ & $0.60 \pm 0.13$ & -0.41 & \\
\hline \multirow[t]{8}{*}{1993.96} & 18 & $\mathrm{CO}$ & 1.2600 .002 & 0.0 & 0.0 & $1.39 \pm 0.01$ & $0.32 \pm 0.01$ & $88.4 \pm 0.1$ & Preuss et al. (1996) \\
\hline & & $\mathrm{C} 1$ & $0.118 \pm 0.004$ & $2.38 \pm 0.02$ & $-90.92 \pm 0.28$ & $1.75 \pm 0.05$ & 0 & $14.45 \pm 0.79$ & VLBA \\
\hline & & $\mathrm{C} 2$ & $0.197 \pm 0.006$ & $5.44 \pm 0.11$ & $-95.82 \pm 0.06$ & $6.35 \pm 0.23$ & $0.37 \pm 0.02$ & $85.86 \pm 0.31$ & \\
\hline & & $\mathrm{C} 3$ & $0.049 \pm 0.003$ & $12.26 \pm 0.35$ & $-102.12 \pm 0.61$ & $12.85 \pm 1.86$ & $0.59 \pm 0.10$ & $-45.47 \pm 7.98$ & \\
\hline & & $\mathrm{C} 4$ & $0.081 \pm 0.001$ & $25.62 \pm 0.01$ & $-103.08 \pm 0.09$ & $8.77 \pm 0.03$ & $0.31 \pm 0.01$ & $-23.66 \pm 0.12$ & \\
\hline & & $\mathrm{C} 5$ & $0.145 \pm 0.005$ & $30.09 \pm 0.04$ & $-107.99 \pm 0.05$ & $12.94 \pm 0.31$ & $0.80 \pm 0.01$ & $-70.78 \pm 0.44$ & \\
\hline & & $\mathrm{C} 4 / 5$ & …................. & 28.49 & 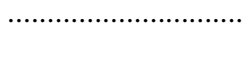 & $\ldots \ldots \ldots \ldots$ & $\ldots \ldots \ldots \ldots \ldots \ldots$ & $\cdots \cdots$ & \\
\hline & & C6 & $0.117 \pm 0.007$ & $38.24 \pm 0.93$ & $-88.42 \pm 1.11$ & $35.22 \pm 0.84$ & $0.73 \pm 0.02$ & $\begin{array}{c}\ldots \ldots \ldots \ldots \ldots . . \\
-55.27 \pm 4.86\end{array}$ & \\
\hline \multirow[t]{8}{*}{1995.79} & 18 & $\mathrm{D}$ & $1.070 \pm 0.10$ & 0.0 & 0.0 & & & & Aaron et al. (1996) \\
\hline & & $\mathrm{C} 1$ & $0.369 \pm 0.04$ & $1.98 \pm 0.3$ & $-92.5 \pm 4.6$ & & & & VLBA \\
\hline & & $\mathrm{C} 2$ & $0.097 \pm 0.01$ & $6.31 \pm 0.5$ & $-94.6 \pm 4.7$ & & & & \\
\hline & & $\mathrm{C} 3$ & $0.039 \pm 0.01$ & $10.44 \pm 0.6$ & $-94.8 \pm 4.7$ & & & & \\
\hline & & $\mathrm{C} 4$ & $0.029 \pm 0.01$ & $26.47 \pm 1.2$ & $-103.7 \pm 5.2$ & & & & \\
\hline & & $\mathrm{C} 5$ & $0.273 \pm 0.03$ & $28.97 \pm 1.0$ & $-104.7 \pm 5.2$ & & & & \\
\hline & & $\mathrm{C} 4 / 5$ & & 28.73 & & & & & \\
\hline & & & ……........... & ……...... & 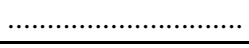 & ….......... & ․․……....... & ……....... & \\
\hline \multirow[t]{8}{*}{1999.68} & 6 & $\mathrm{~A}$ & $1.689 \pm 0.169$ & 0.00 & $-90.00 \pm 5.00$ & 0.21 & 1 & & this paper \\
\hline & & A1 & $0.399 \pm 0.040$ & $0.71 \pm 0.20$ & $-89.82 \pm 5.00$ & 0.26 & 1 & & VLBA \\
\hline & & $\mathrm{A} 2$ & $0.277 \pm 0.028$ & $1.48 \pm 0.20$ & $-90.08 \pm 5.00$ & 0.53 & 1 & & \\
\hline & & A3 & $0.039 \pm 0.004$ & $2.07 \pm 0.20$ & $-90.36 \pm 5.00$ & 0.98 & 1 & & \\
\hline & & A4 & $0.080 \pm 0.008$ & $3.33 \pm 0.20$ & $-90.17 \pm 5.00$ & 2.01 & 1 & & \\
\hline & & A5 & $0.030 \pm 0.003$ & $5.81 \pm 0.20$ & $-90.93 \pm 5.00$ & 2.43 & 1 & & \\
\hline & & A6 & $0.049 \pm 0.005$ & $8.57 \pm 0.20$ & $-90.73 \pm 5.00$ & 2.94 & 1 & & \\
\hline & & A7 & $0.105 \pm 0.011$ & $28.07 \pm 1.00$ & $-95.39 \pm 5.00$ & 10.57 & 1 & & \\
\hline
\end{tabular}


Table 2. Linear regression performed on the core separation versus time relation (see Fig. 7).

\begin{tabular}{ccrr}
\hline \hline Jet component at & \multicolumn{1}{c}{$A_{0}$ [mas] } & $A_{1}$ [mas/year] & \multicolumn{1}{c}{$\beta_{\text {app }}$} \\
\hline 6 mas & $31.685 \pm 62.230$ & $-0.013 \pm 0.031$ & $-0.4 \pm 1.0 c$ \\
12 mas & $297.015 \pm 182.774$ & $-0.144 \pm 0.092$ & $-4.8 \pm 3.1 c$ \\
I) 25 mas ("one to one") & $-283.504 \pm 142.542$ & $0.155 \pm 0.072$ & $5.2 \pm 2.4 c$ \\
II) 25 mas ("one to two") & $-364.516 \pm 19.829$ & $0.196 \pm 0.010$ & $6.5 \pm 0.3 c$ \\
III) 25 mas ("one to two") & $-399.158 \pm 98.468$ & $0.214 \pm 0.049$ & $7.1 \pm 1.7 c$ \\
\hline
\end{tabular}

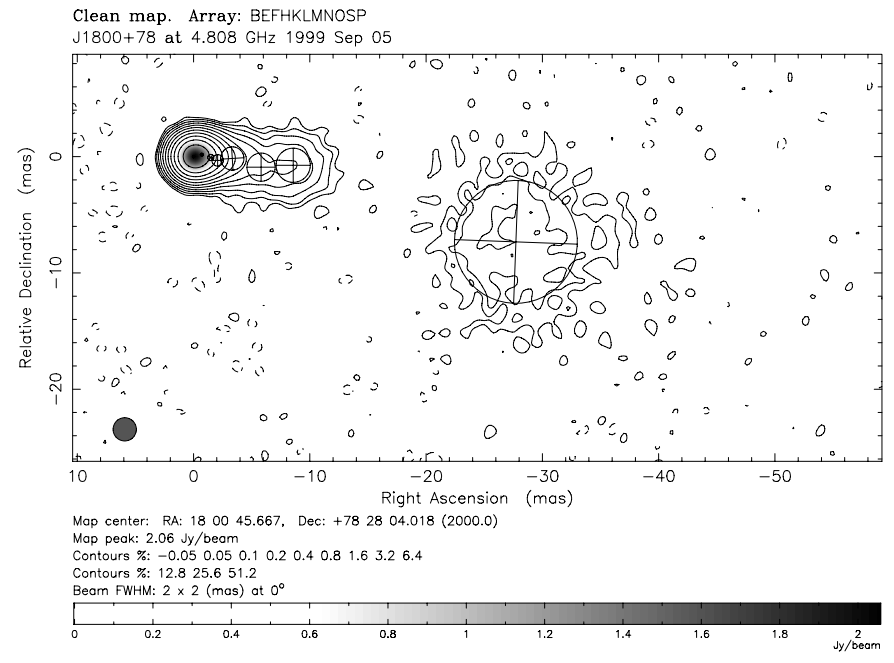

Fig. 6. An example for a model fit (performed with the $5 \mathrm{GHz}$ data from 1999.68) is shown.

represent the most likely scenario. The core separation of the flux-density weighted "one-to-two" 25 mas components is given for the three epochs 1993.41, 1993.96, and 1995.79 between the two horizontal dotted lines (Table 1).

In Fig. 7 we show the core separation versus time for the components within 30 mas of the core. We indicate the three different identification scenarios for the $r \sim 25$ mas component by different line styles. In Table 2 we give the parameters of a linear regression performed on the core separation versus time for the different jet components and list the calculated apparent velocities for the different identification scenarios respectively. The error of the velocity is the standard error of the slope of the performed regression. We find almost no motion for the component at 6 mas. Thus this component might be stationary. The negative apparent motion of the component at 12 mas can be explained by a blend of two individual jet components that have repeatedly been observed at values of $\sim 10$ and $\sim 12$ mas. Further observations - more densely spaced in time - will clarify whether this component is single or double. We find values between $5.2 c( \pm 2.4 c)$ and $7.1 c( \pm 1.7 c)$ for the 25 mas component. Based on these calculations we tentatively suggest that this component reveals apparent superluminal motion on the basis of observations spanning almost 18 years.

Figure 8 shows the flux-density of the different jet components within 30 mas of the core. Significant flux-density variability is found for the core; however, the different frequency of the $5 \mathrm{GHz}$ data point has to be taken into account.

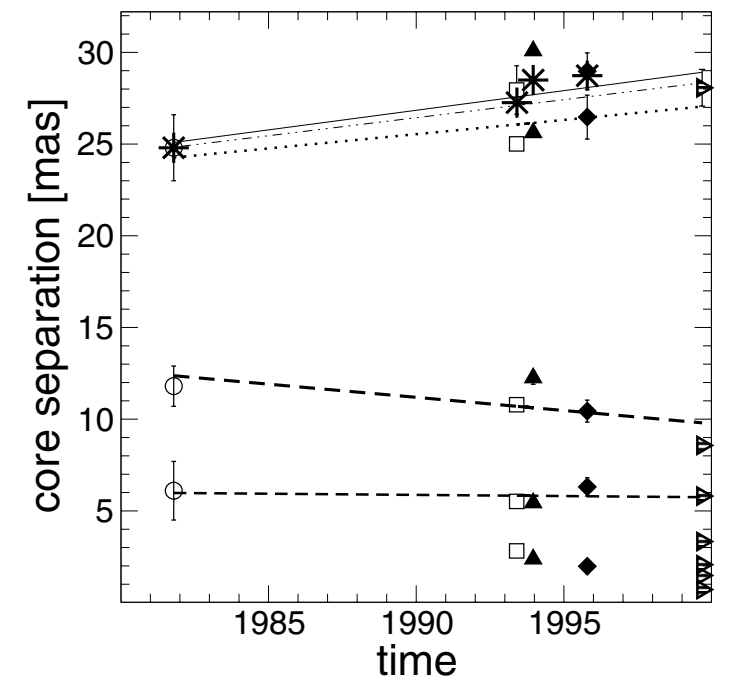

Fig. 7. Core separation versus time for components within 30 mas core separation. The lines indicate the linear regressions performed for the motion of the jet components. In the case of the $r \sim 25$ mas component three scenarios are possible (for details, see text) and indicated here by different line styles ("one-to-one": dotted line, "one-to-two": dot-dashed line). The regression for the third (flux-density weighted) scenario is shown as a solid line, the values are plotted as stars.

In Fig. 9 we show the position angle versus core separation for these same jet components. Different symbols denote different epochs (see Fig. 8). The data points of the $6 \mathrm{~cm}$ observations appear at slightly less negative position angles than the $18 \mathrm{~cm}$ data indicating frequency dependent position shifts.

\section{Discussion}

S5 $1803+784$ belongs to a small sample of AGN which have been well-studied with interferometric techniques (e.g., Zensus 1997). Nevertheless, the connection between the pcand kpc-scale jet remained unclear for quite some time. The observations presented in this paper reveal previously unknown peculiarities of the jet of this source. We here discuss the misalignment and properties such as the curvature on all scales, the evidence for jet motion even on large scales, the selfsimilarity over large scales, and the possible evidence for a halo/counter-jet.

\subsection{Self-Similarity: wiggling on large scales}

The jet of $1803+784$ reveals the same basic structure at wavelengths between $7 \mathrm{~mm}$ and $18 \mathrm{~cm}$; we find evidence for 


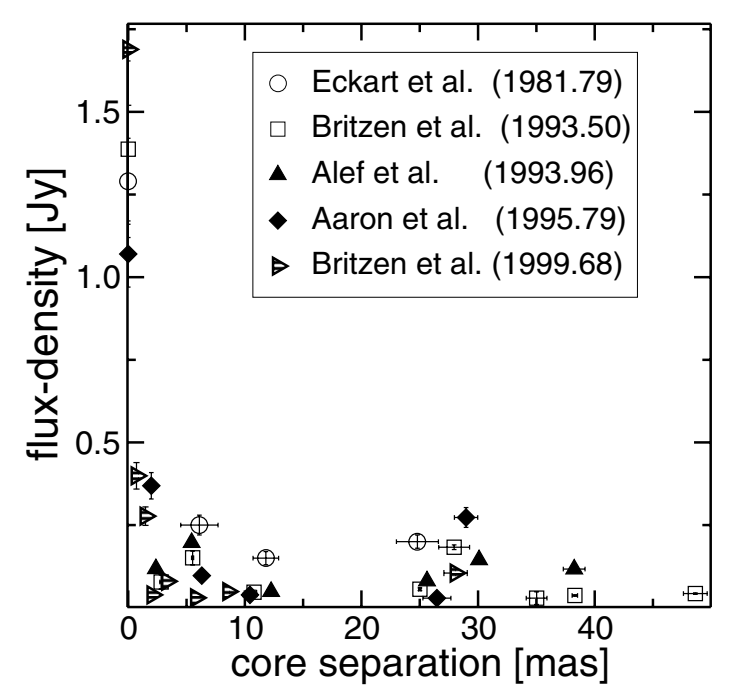

Fig. 8. Flux-density evolution for the components within 30 mas core separation. Different symbols denote different epochs.

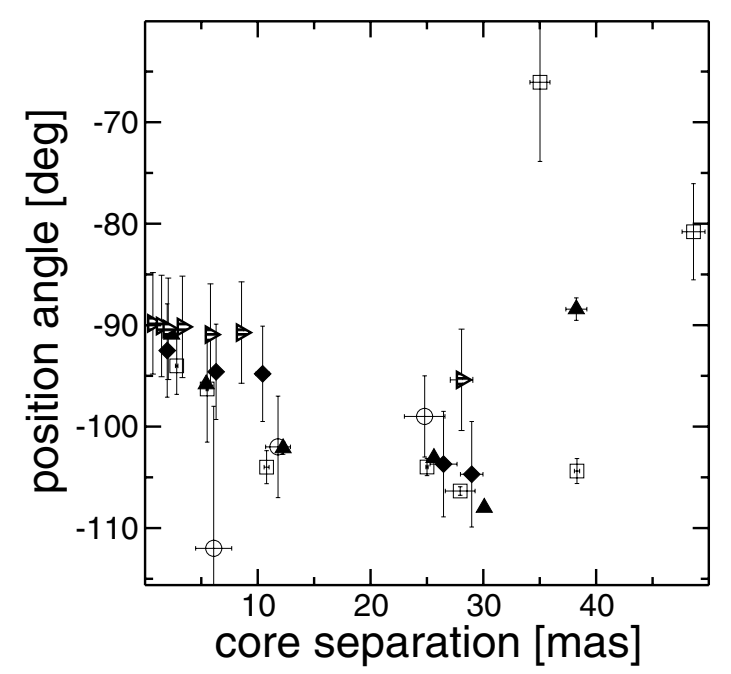

Fig. 9. Position angle versus core separation for components within 30 mas core separation. The symbols for the different components are the same as in Fig. 8.

wiggling on all scales between tenths of a milliarcsecond (e.g., Krichbaum et al. 1994; Britzen et al. 2005) and 50 arcsec (Fig. 5). With the help of the maps presented in this paper (see Figs. 3-5) we show the details of the curved jet-structure on scales between $\sim 30$ mas and 50 arcsec which have not been probed before. The wiggling is directed in an east-west direction up to a core separation of $\sim 0.5 \operatorname{arcsec}$, it then continues in a north-south direction (see Figs. 4 and 5). The jet-bending at a core separation of $\sim 0.5$ arcsec changes the direction of the large scale jet. The change in the propagation direction does obviously not influence the wiggling of the jet.

Jet curvature is often a pronounced phenomenon in higher frequency observations of AGN (e.g., PKS 0420-014, Britzen et al. 2000). The observations taken with the world-array support a model in which the wiggling continues into the kpc-structure.

In addition to the wiggling jet structure, we find a dominant double-structure in the jet. High-frequency observations by Krichbaum et al. (1994) reveal a double structure embedded in a wiggling jet in the vicinity of the core. Observations at $3.6 \mathrm{~cm}$ (Britzen et al. 2005, and references therein) confirm this double structure (separation of $\sim 1.4$ mas) within an overall sinusoidal jet structure extending to core separations of up to 12 mas. The global map obtained in the $18 \mathrm{~cm}$ observations (Fig. 3a) shows a double morphology (core $+r \sim 25$ mas component) and evidence for significant jet curvature. The observed structures (wiggling + double morphology) could be explained within a model of a spatially curved structure, where the superposition of the curved structure and the occasional changes of the jet direction could lead, via Doppler boosting, to brightening and weakening of the jet as observed.

Falle (1991) shows that the flow caused by a supersonic gas jet can be self-similar under the condition that the external density decreases more slowly than $1 / R^{2}$. In this case the global expansion of the flow is approximately self-similar.

\subsection{Motion visible also in the intermediate-scale structure?}

$1803+784$ has been observed at $\lambda=18 \mathrm{~cm}$ with VLBI by several groups in the past. These datasets in combination with our data at 18 and $6 \mathrm{~cm}$ presented in this paper enable us to investigate the jet for motion. The intermediate scale jet reveals remarkable fine scale structure: we find five jet components between 2 and 30 mas (see Table 1), but there is evidence for more components that cannot be resolved with earth-based arrays. Additional observations with HALCA have already been performed and will soon clarify this point.

While a stationary jet component at $\sim 1.4$ mas core separation has been claimed by several authors, superluminal motion has as well been observed at mm-wavelengths and in observations at $3.6 \mathrm{~cm}$ (Britzen et al. 2005) for jet components at core separations smaller than $\sim 1.4$ mas. Britzen et al. (2005) present an alternative model to the stationarity of the 1.4 mas component based on oscillations found in the distance between the core and this component. Reconfinement shocks are capable of reproducing the observed behaviour.

The position of the jet component at $\sim 6$ mas core separation has been confirmed by several authors and no significant change in position has so far been observed. From our observations spanning 14 years in time we conclude that this component does not show motion: the apparent velocity according to our calculations is $-0.4 c( \pm 1.0 c)$.

The component at 12 mas core separation seems to approach the core based on our observations. However, our observations (see Table 1) also indicate that this component might be a blend of two components at core separations of $\sim 10$ and $\sim 12$ mas respectively. Thus, we might have identified two individual components as one. A paper decribing the motion and stationarity of jet components in $1803+784$ for the inner 12 mas of core separation is in preparation.

In this paper we have also calculated the apparent velocities based on data obtained at two frequencies, namely 1.6 and $5 \mathrm{GHz}$. Possible frequency-dependent position shifts might influence the apparent velocity calculations. Further higher 
dynamic range observations and also comparisons with other different-frequency data might clarify this point.

The motion studies are handicapped by the limited resolution of the ground-array observations: different identification scenarios for the 25 mas component cannot be cleared up. We present three different scenarios for the identification of the 25 mas component across the epochs. Assuming that the 25 mas component seen by Eckart et al. (1986) can be resolved into two components in the following years, we find values for the apparent motion between $\sim 6.5$ and $\sim 7 c$ depending on whether the position of the resulting component is fluxdensity weighted or not. The flux-density weighted position seems to yield the most reliable result.

We thus derive a complex motion scenario: some components seem to be stationary $\left(\beta_{\text {app }}<c\right.$; e.g., component at 6 mas core separation) but superluminal at 25 mas core separation (between $\sim 5$ and $\sim 7 c$ ). The coexistence of almost stationary and moving components as discussed for $1803+784$ in this paper can be explained within relativistic time-dependent hydrodynamical models (e.g., Agudo et al. 2001) as a result of the interaction of the superluminal component with the underlying jet. Multiple conical shocks form behind the main perturbation. Those appearing closer to the core could be identified as stationary components.

A different scenario has been suggested for 4C 39.25, the most prominent example known for the simultaneous existence of stationary and moving components. Alberdi et al. (2000) interpret the stationary feature in 4C 39.25 as a bend in the jet trajectory in a plane that does not contain the observer, and the moving one as a shock turning around the bend. This scenario ist most probably not applicable to $1803+784$ since the moving components separate from the core and no motion on the pc-scale counter-jet side is found.

\subsection{Halo visible in $1803+784$ ?}

The morphology of the WSRT map suggests two hot spots at the end of a curved and spatially bent jet. The nature of the halo around the core is still unclear and will have to be investigated further. Is it real, or just an artefact fom imaging and point-source subtraction? If real, we might interpret the halo as part of a more extended counter-jet lobe seen from the "back" relative to its motion. However then we have the problem of why we don't see a similar lobe on the jet side.

\subsection{Misalignment}

Many AGN radio (and optical) jets reveal curved jet structures (e.g., Zensus 1997) and distortions of the jet path produced by various mechanisms are common features of radioloud AGN. It is generally believed that the small-scale jets observed in VLBI images are the sources of, and are continuous with, the large scale jets that supply the outer lobes. $1803+784$ belongs to a group of 20 intriguing AGN forming the so-called misaligned population characterized by a difference in the projected position angle between the pc-kpc scale jets of $70^{\circ}$ to $90^{\circ}$ (referred to as "secondary peak" of the distribution of VLBI-VLA jet $\triangle \mathrm{PA}$ by Appl et al. 1996).

According to Appl et al. (1996), no significant correlation has been found between morphological criteria (e.g., wide range of apparent types) and other properties (e.g., variability, apparent motion, ratio of core to extended flux) of misaligned sources in general. They suggest that one common feature across misaligned sources seems to be the obvious "helical" form of the jets.

A few other misaligned objects have been studied in detail in the literature and are particularly well mapped at the intermediate scale of 10 to 100 mas with MERLIN or the European VLBI Network (e.g., 3C 216, 3C 309.1, 3C 345, and Mkn501).

Rather than constituting a homogeneous sample, they all show individual peculiarities.

In the case of 3C 345, an archetypical source whose VLBI jet components follow different "quasi-helical 3-dimensionally bent trajectories" (Kollgaard et al. 1989), two outer knots aligned with the nucleus suggest a sudden change in the orientation of the central engine.

For most other misaligned sources, the relation between the pc-scale and kpc-scale structure is not known since we don't see the big bend.

Several solutions to the misalignment problem in 1803+784 have been discussed (see Strom \& Biermann 1991). Tateyama et al. (2002) discuss the overall radio morphology of $1803+784$ as described by two dominant components: a narrow helical jet of $6 \mathrm{kpc}$ in extension and a much larger north-south component perpendicular to the jet as a "dogleg" structure. They attribute the "dogleg" structure to a collision between the jet and a massive cloud (Stocke et al. 1985).

The most simple solution would be that the southern component in the VLA image by Antonucci et al. (1986) belongs to another radio source. This can be excluded on the basis of the bridge between the core and the southern component found by Strom \& Biermann (1991) and the continuous jet-structure seen in the WSRT- and MERLIN maps presented in this paper (Figs. 5 and 4). The continuation of the wiggling jet over large scales towards the southern component in the WSRT and MERLIN observations supports the idea that the southern component is part of $1803+784$.

Two main types of mechanisms for the deformation of jets from a "straight" path can be distinguished, namely interaction of the flow with the ambient medium, and a change of orientation of the jet source. The former comprise bending by ram pressure, pressure gradients (e.g., Fiedler \& Henriksen 1984) and Kelvin-Helmholtz instabilities (e.g., Hardee 1982, 2003).

A change of orientation of the jet source can be caused by the precession of the axis of the central black hole or of the accretion disc, the misalignment between the rotation axes of the accretion disk and of the Kerr black hole (spin-induced precession, Caproni et al. 2004), and the sweeping magnetic twist mechanism (Nakamura et al. 2001). Binary black holes might play an important role in this (e.g., Begelman et al. 1980; Britzen et al. 2000, 2001; Conway \& Murphy 1993). Several authors have argued that jets that are initially straight and pressure-confined should naturally develop helical distortions due to the effects of Kelvin-Helmholtz instabilities (e.g., 
Hardee 1982, 2003). A model explaining how such instabilities can give rise to the observed wiggling on different scales has been presented by Conway \& Murphy (1993) and Conway \& Wrobel (1995), the latter applied to Mkn 501. Appl et al. (1996) suggested a model for the second mechanism: twisted accretion discs, which should be even more frequent than binary black holes in the central engines of active galactic nuclei.

$1803+784$, as discussed before, reveals apparent superluminal motion of jet components on different scales in addition to stationary or quasi-stationary components (Britzen et al. 2005 , this paper). Even at $\sim 25$ mas, as suggested in this paper, we find some indication for apparent superluminal motion. In addition, the linear propagation of the jet is perturbed from the inner pc- to kpc-scales. If the ridge-line of components traces the jet, then oscillations continue even on scales of up to $100 \mathrm{kpc}$. The existence of bends of as much as $90^{\circ}$ (in projection) on scales extending over three orders of magnitude suggests an intrinsic origin in the jet itself (e.g., change in the orientation of the central engine), although higher sensitivity observations are required to verify this on the largest dimensions by mapping the more diffuse emission. Because the relativistic jets of blazars, such as $1803+784$, are thought to be pointing at a small angle to the observer, small intrinsic bends are strongly enhanced by projection.

\subsection{On the nature of the secondary component}

Based on the spectral decomposition of Strom \& Biermann (1991), the spectrum of the southern hot spot is expected to show a turnover at low frequencies. This turnover frequency can be estimated using the formula for the magnetic field $(B)$ from synchrotron self absorption. Requiring $B$ to be in the range of $10^{-6}<B<1$ Gauss, the turnover should be observable in the range of $2 \mathrm{MHz}<v_{m}<15 \mathrm{MHz}$. This requirement limits the flux to be $S_{m}\left(v_{m}\right)=1-2$ Jy for the hot spot at this frequency.

The so-called critical size can be determined via the two expressions for the magnetic energy from synchrotron self $a b-$ sorption theory and from equipartition arguments. A rough calculation yields for the critical size in our case: $\theta_{\text {crit }}$ [mas] $=$ $5.7 v_{m}$, where $v_{m}$ is the turnover frequency in GHz. For this calculation, we further assume a luminosity distance of $3.52 \mathrm{Gpc}$ and a turnover flux-density of $1 \mathrm{Jy}$. We obtain for $v_{m}=2 \mathrm{MHz}$ a critical size of $\theta_{\text {crit }}=2800 \mathrm{mas}$, and for $v_{m}=15 \mathrm{MHz}$ a critical size of $\theta_{\text {crit }}=377$ mas. The size of the compact component at $r=45^{\prime \prime}$ can be measured (from the map) to be about 5000 mas. Thus the hot spot is much larger than $\theta_{\text {crit }}$, and therefore is most likely out of equipartition, dominated by magnetic field energy. This is consistent with the hypothesis of a bow shock (or working surface) that propagates into the surrounding IGM. The important role magnetic fields are playing in the bow shock will have to be investigated in more detail.

It is interesting to note that for $v_{m}=3 \mathrm{MHz}$, and for a size of 5000 mas, the magnetic energy density is equal to that of the CMB (Miley 1980), which is about $11 \mu \mathrm{G}$ at a redshift of 0.68 .

\section{Conclusions}

With the help of WSRT observations performed at $6 \mathrm{~cm}$, VLBA observations at $6 \mathrm{~cm}$, and world-array observations including MERLIN performed at $18 \mathrm{~cm}$, we have probed the structure of $1803+784$ on different angular scales. At a core separation of $\sim 0.5$ arcsec the MERLIN-only image reveals a 90 degree bending of the jet towards the South (see Fig. 4) and the WSRT observations trace this jet further towards the southern component. This confirms the southern component at $\sim 45^{\prime \prime}$ core separation as a jet component belonging to S5 $1803+784$.

Curved jet structure is visible in S5 $1803+784$ even on large scales (arcseconds). The observations taken with WSRT and the MERLIN array support a model in which the wiggling and probably even helicity continues into the kpc-structure.

We possibly find evidence for a halo on the counter-jet side in the WSRT observations.

We find some indication for apparent superluminal motion of the 25 mas jet component. Three different scenarios yield a value between $\sim 5$ and $\sim 7 c$. In addition, we find evidence for the coexistence of apparently stationary and superluminal components in the intermediate scale jet.

HALCA observations have already been performed, and will clarify the correct identification scenario and will yield further constraints on the apparent motion.

Acknowledgements. We are particularly grateful to C. Schalinski for extensive discussions and helpful suggestions on the different subjects of this paper. We thank S. Aaron for permission to reproduce material. We thank S. Unwin for careful supervising of the correlation. We thank the $2 \mathrm{~cm}$ collaboration for providing the dataset. This work was supported by the European Comission, TMR Programme, Research Network Contract ERBFMRXCT96-0034 "CERES" and by the DLR, project 50QD0101. S. Britzen acknowledges support by the ClaussenSimon Stiftung. The WSRT is operated by ASTRON with financial support from the Netherlands Organisation for Scientific Research (NWO). This research has made use of the NASA/IPAC Extragalactic Database (NED) which is operated by the Jet Propulsion Laboratory, California Institute of Technology, under contract with the National Aeronautics and Space Administration.

\section{References}

Aaron, S. E. 1996, Ph.D. Thesis, Brandeis University

Agudo, I., Gómez, J.-L., Martí, J.-M., et al. 2001, ApJ, 549, L183

Alberdi, A., Gómez, J.-L., Marcaide, J. M., et al. 2000, A\&A, 361, 529

Antonucci, R. R. J., Hickson, P., Olszewski, E. W., \& Miller, J. S. 1986, AJ, 92, 1

Antonucci, R. R. J., Hickson, P., Miller, J. S., \& Olszewski, E. W. 1987, AJ, 93, 785

Appl, S., Sol, H., \& Vicente, L. 1996, A\&A, 310, 419

Begelman, M. C., Blandford, R. D., \& Rees, M. J. 1980, Nature, 287, 307

Benson, J. M., Walker, R. C., Unwin, S. C., et al. 1988, ApJ, 334, 560

Biermann, P. L., Schaaf, R., Pietsch, W., et al. 1992, A\&AS, 96, 339

Britzen, S., \& Krichbaum, T. P. 1995, in Proc. of the 10th Working meeting on European VLBI for Geodesy and Astrometry, ed. R. Lanotte, \& G. Bianco, Centro Di Geodesia Spaziale, Matera, Italy, 172

Britzen, S., Witzel, A., Krichbaum, T. P., et al. 1999, NewAR, 43, 751 
Britzen, S., Witzel, A., Krichbaum, T. P., et al. 2000, A\&A, 360, 65

Britzen, S., Roland, J., Laskar, J., \& Kootas, K. 2001, A\&A, 374, 784 Britzen, S. 2002, Rev. Mod. Astron., 15, 199

Britzen, S., Witzel, A., Krichbaum, T. P., et al. 2005, MNRAS, in press Caproni, A., Mosquera Cuesta, H. J., \& Abraham, Z. 2004, ApJ, 616, 99

Cawthorne, T. V., Wardle, J. F. C., Roberts, D. H., et al. 1993, ApJ, 416, 496

Charlot, P. 1990, A\&A, 229, 51

Conway, J. E., \& Murphy, D. W. 1993, ApJ, 411, 89

Conway, J. E., \& Wrobel, J. M. 1995, ApJ, 439, 98

Eckart, A., Witzel, A., Biermann, P., et al. 1986, A\&A, 168, 17

Eckart, A., Witzel, A., Biermann, P., et al. 1987, A\&A, 67, 121

Falle, S. A. E. G. 1991, MNRAS, 250, 581

Fey, A. L., Clegg, A. W., \& Fomalont, E. B. 1996, ApJS, 105, 299

Fiedler, R., \& Henriksen, R. N. 1984, ApJ, 281, 554

Gabuzda, D. C. 1999, NewAR, 43, 691

Gabuzda, D. C., \& Cawthorne, T. V. 2000, MNRAS, 319, 1056

Gabuzda, D. C., \& Chernetskii, V. A. 2003, MNRAS, 339, 669

Hardee, P. E. 1982, ApJ, 257, 509

Hardee, P. E. 2003, ApJ, 597, 798

Hummel, C. A., Muxlow, T. W. B., Krichbaum, T. P., et al. 1992, A\&A, 266, 93

Johnston, K. J., Biermann, P., Eckart, A., Witzel, A., et al. 1984, ApJ, 280,542

Kellermann, K. I., Vermeulen, R. C., \& Zensus, J. A. 1998, AJ, 115, 1295

Kollgaard, R. I., Wardle, J. F. C., \& Roberts, D. H. 1989, AJ, 97, 1550

Kollgaard, R. I., Wardle, J. F. C., Roberts, D. H., \& Gabuzda, D. C. 1992, AJ, 104, 1687

Krichbaum, T. P. 1990, in Parsec-scale Radio Jets, ed. J. A. Zensus, \& T. J. Pearson (Cambridge: Cambridge University Press), 83

Krichbaum, T. P., Witzel, A., Graham, D. A., et al. 1993, in Subarcsecond Radio Astronomy, ed. R. J. Davies, \& R. S. Booth (Cambridge: Cambridge University Press), 181

Krichbaum, T. P., Standke, K. J., Graham, D. A., et al. 1994, IAU Symp., 159, 187
Lawrence, C. R., Readhead, A. C. S., Pearson, T. J., \& Unwin, S. C. 1987, in Superluminal Radio Sources, ed. J. A. Zensus, \& T. J. Pearson (Cambridge: Cambridge University Press), 260

Miley, G. 1980, ARA\&A, 18, 165

Muxlow, T. W. B., Junor, W., \& Spencer, R. E. 1988, in Proc. IAU Symp. ed. M. J. Reid, \& J. M. Moran (Dordrecht: Kluwer), 129, 131

Nakamura, M., Uchida, Y., \& Hirose, S. 2001, NewA, 6, 61

Pearson, T. J., \& Readhead, A. C. S. 1988, ApJ, 328, 114

Preuss, E., Alef, W., \& Kellermann, K. I. 1996, in Proc. IAU Symp., ed. R. D. Ekers, C. Fanti, \& L. Padrielli (Dordrecht: Kluwer), 175, 35

Ros, E., Marcaide, J. M., Guirado, J. C., et al. 2000, A\&A, 356, 357

Ros, E., Marcaide, J. M., Guirado, J. C., \& Pérez-Torres, M. A. 2001, A\&A, 376, 1090

Schalinski, C. J., Alef, W., Witzel, A., et al. 1988, in Proc. IAU Symp., ed. M. J. Reid, \& J. M. Moran (Dordrecht: Kluwer), 129, 359

Stickel, M., Padovani, P., Urry, C. M., Fried, J. W., \& Kühr, H. 1991, ApJ, 374, 431

Stickel, M., Fried, J. W., \& Kühr, H. 1993, A\&AS, 98, 393

Stocke, J. T., Burns, J. O., \& Christiansen, W. A. 1985, ApJ, 299, 799

Strom, R. G., \& Biermann, F. L. 1991, A\&A, 242, 313

Tateyama, C. E., Kingham, K. A., Kaufmann, P., \& de Lucena, A. M. P. 2002, ApJ, 573, 496

Wagner, S., Sanchez-Pons, F., Quirrenbach, A., \& Witzel, A. 1990, A\&A, 235, L1

Wagner, S. J., \& Witzel, A. 1995, ARA\&A, 33, 163

Wehrle, A. E., Cohen, M. H., Unwin, S. C., et al. 1992, ApJ, 391, 589

Windhorst, R. A., van Heerde, G. M., \& Katgert, P. 1984, A\&AS, 58, 1

Witzel, A. 1987, in Superluminal Radio Sources, ed. A. Zensus, \& T. J. Pearson (Cambridge: Cambridge University Press), 83

Witzel, A., Schalinski, C. J., Johnston, K. J., et al. 1988, A\&A, 206, 245

Zensus, J. A. 1997, ARA\&A, 35, 607 